\title{
Efficacy and safety of Specific Conjugate Particle (SCP)- Doxorubicinin Patients with Recurrent High-Grade Gliomas, a Randomized Clinical Study
}

\begin{abstract}
Keywords: Glioma; Glioblastoma; SCP-Doxorubicin; PEGDoxorubicin; Phase III trial; Conjugate particle; Adverse events; Efficacy

Abstract

Objective: Doxorubicin has proven to be partly efficacious in treating glioblastoma multiforme. However, the conventional formulation of doxorubicin has not been used clinically, due to poor penetration of the blood-brain barrier.To overcome these obstacles authors compared the use of Specific conjugate particle doxorubicin (Group 1), a proven efficacious agent with advanced technical drug and pegylated liposomal Doxorubicin (Group 2) in a randomized prospective Phase III trial involving patients with recurrent high-grade glioma.
\end{abstract}

Methods: We recruited eighty (80) patients with WHO grade III-IV high-grade glioma, according to 2 independent pathology reports, tumor recurrence identified on gadolinium-enhanced Magnetic Resonance Imaging (MRI). The participants were between the ages of 18 and 70 years, with a life expectancy of more than two months. Patients were randomized to receive either Specific Conjugate Particle Doxorubicin (SCP-Doxorubicin) or the conventionally accepted PEGylated Liposomal Doxorubicin (PEG- Doxorubicin). Study subjects received $20 \mathrm{mg} / \mathrm{m} 2$ of either PEG-Doxorubicin or SCP-Doxorubicin by an intravenous infusion over 30 minutes per day, with a range of 1-28 courses administered per patient.

Results: In the patients evaluated, overall response rate was $40 \%$ is it for both groups, what is the percentage for each group. Two patients achieved Complete Responses (CRs) and two Partial Responses (PRs) in the SCP-Doxorubicin arm. We noted Stable Disease (SD) lasting greater than eight weeks in 28 patients. Patients receiving SCPDoxorubicin had a significantly better response to therapy (more CR, $P R$, and $S D$ ) than those receiving PEG-Doxorubicin $(p<0.05)$. Patients had also a significantly better survival (more PFS6, TTP and OS) to SCPDoxorubicin therapy than those receiving PEG- Doxorubicin $(p<0.05)$ $\{$ where is the PFS6, TTP, OS\}. Twenty-five adverse events occurred in patients receiving PEG-Doxorubicin, whereas there were only fou adverse events in patients who received SCP-Doxorubicin. Palmar Plantar Erythrodysesthesi (PPE) was the most common adverse event in both the groups $(p<0.05)$.

Conclusion: SCP-Doxorubicin had superior efficacy when compared to PEG-Doxorubicin independent of the patient's prio therapeutic regime and stage of carcinoma. Furthermore, SCPDoxorubicin was found to be a comparatively safer treatment regimen with no major side effects and a significantly lower adverse event rate than PEG- Doxorubicin.

\section{Abbrevations}

SCP: Specific Conjugate Particle; PEG-Dox: Pegylated Liposomal Doxorubicin; SCP-DOX: Specific Conjugate Particle Doxorubicin; PFS: Progression Free Survival; OS: Overall Survival; TTP: Time to

\section{Journal of}

Vaccine \&

\section{Immunotechnology}

\section{Timothy Allen ${ }^{1 *}$, Nepton Sheik Khoni ${ }^{2}$, Bruce Dunphy ${ }^{3}$, and Naveed Basha Court ${ }^{4}$}

${ }^{\text {I} G l o b a l}$ Allied Pharmaceuticals, Center for Excellence in Research \& Development, USA

${ }^{2}$ Global Allied Pharmaceutical, Center for Excellence in Research \& Development, USA

${ }^{3}$ School of Public Health and Social Work, Australia

${ }^{4}$ Global Allied Pharmaceuticals, Center for Excellence in Research \& Development, USA

\section{Address for Correspondence}

Timothy Allen, Global Allied Pharmaceuticals, Center for Excellence in Research \& Development, 160 Vista Oak Dr. Longwood, FL 32779 , USA, Tel: 1-321-945-4283; E-mail: timothy.allen@gapsos.com

Submission: 01 March, 2017

Accepted: 03 April, 2017

Published: 10 April, 2017

Copyright: (c) 2017 Allen T, et al. This is an open access article distributed under the Creative Commons Attribution License, which permits unrestricted use, distribution, and reproduction in any medium, provided the original work is properly cited.

Progression (TTP); CR: Complete Repsonse; PR: Partial Response; SD: Stable Disease; MRI: Magnetic Resonance Imaging; WHO: World Health Organization

\section{Introduction}

High-grade gliomas are the most common malignant brain tumors and have a poor prognosis. Glioblastoma is the most frequently diagnosed and constitutes $16 \%$ of all brain tumors[1]. Surgery is the initial treatment of high-grade glioma which usually involves removing as much of the tumor as safely possible. The goals of surgery are to obtain a diagnosis, alleviate symptoms related to increased intracranial pressure or compression, increase survival, and decrease the need for corticosteroids. The median survival with surgery alone is approximately 4 months. Radiation is standard therapy for patients with high-grade gliomas after either maximal excision or biopsy. When used in combination with radiation therapy and surgery, chemotherapy may improve survival and quality of life in some patients with high-grade gliomas. The drug that are most widely used for high-grade glioma include temozolomide [2]. There was an absolute increase in 1-year survival from $40 \%$ to $46 \%$ and a 2 -month increase in median survival when chemotherapy was added to postoperative radiation [3].

Therapeutic advances have been made in the past decade with the addition of temozolomide chemotherapy to maximal safe tumor resection and radiotherapy. However, median survival is still limited to only 15 months. Therefore, novel therapies are urgently needed $[4,5]$.

Doxorubicin is one of the most effective agents in vitro against cell lines derived from malignant glioma [6]. However, it has no significant effect in vivo, and this finding appears to be attributed primarily to poor blood-brain barrier penetration. In a tumor model, 
tissue and cerebrospinal fluid concentrations of doxorubicin increased when sterically stabilized liposomes were used [7]. Concentrations were further elevated with the use of the PEGylated formulation of doxorubicin (PEG-DOX) [8].

Doxorubicin is also known to induce tumor resistance genes. Doxorubicin is known to induce expression of members of the adenosine tri phosphate-binding super family of transporter proteins, such as Multi Drug Resistance protein 1 (MDR-1) [9] and Multiple Resistance Protein (MRP) [10]. These are known to play major roles in the development of cellular resistance to chemotherapeutic agents [11]. MRP is strongly expressed in up to $70 \%$ of central nervous system tumour specimens, and MDR-1 expression has been detected in $18 \%$ of high-grade gliomas [12].

In other words, our current indulgence of the molecular basis of cancer, the progress in its discovery and treatment, and its high mortality is acute in seeking the right cure despite great advances have been made in therapies. SCP- Dox is an attempt in proceeding with the current treatment regimens for cancer which have shown limited survival benefits when used for most advanced stage cancers. We generally target the treatments on tumor bulk but not its cancer stem cells $[13,14]$. Conventional therapies target cancer cells which are highly proliferative and improve the patients survival if properly addressed/targeted [15]. The traditional cancer therapies, including surgery, hormonal therapy, anti-angiogenesis therapy, and/or immunotherapy show the lack of prolonged efficacy in its long- term outcome. This is deemed to the non- specific effects on normal cells. SCP-Dox may be deemed as an opening in answering this important element of our fight against cancer cells and/or its neoplastic tissues. We tackle the tumors specifically by utilizing leaky tumor phenomenon of the targeted malignancies and expose the stem cells in addition to the differentiating cells. This allows us to venture into the longer overall survival among the treated patients with the highly aggressive tumors such as STS, GBM, etc. We use the particle producing element of the SCP-Dox to mimic salinomycin, sulforaphane, a novel Gemini vitamin D analog (BXL0124) and so on. These naturally occurring compounds have the ability to target the stem cells which in turn relinquish the element of their refractory nature of the neoplasm [16-19]. We know that cancer stem cells possess characteristics associated with normal stem cells, specifically the ability to give rise to all cell types found in a particular cancer sample; however, it is often considered to be associated with chemo resistance and radio resistance that lead to the failure of traditional therapy [20]. Hence a new technology was invented to stabilize the Doxorubicin better called "Specific Conjugate Particle" (SCP) technology; we combined two different and independently acting compounds into one hybrid compound that can synergize. The potency of the new composite compound is greater than the sum of each moiety. The precedent hybrid compound comes from naturally occurring proteins and small molecules, such as botulinum toxin and bleomycin [21].

The SCP-Doxorubicin is 4-5 times more soluble in water and no precipitation or sedimentation was observed even after 24 hours of dissolving it in water at room temperature when compared to conventional doxorubicin. The solution remains stable over a wide $\mathrm{pH}$ range, with no aggregation at either acidic or neutral $\mathrm{pH}$. The hybrid formulation is stable for two years in cold $\left(8^{\circ} \mathrm{C}\right)$ and for four weeks at $37^{\circ} \mathrm{C}$. There is no sign of interaction of serum proteins upon entry of the hybrid product into the body. Based on the available findings, we initiated a clinical trial which involved the use of SCPDOX (Group 1) versus PEG-DOX (Group 2) to treat patients with recurrent high-grade glioma.

\section{Materials and Methods \\ Patients and selection criteria}

Protocols were drafted by standard good clinical practice guidelines and with the Helsinki Declaration. The local human investigations committee had approved both protocols before they were initiated, and informed consent was obtained from each patient before enrollment. The major eligibility criteria were high-grade glioma (World Health Organization [WHO] Grade III-IV) according to 2 independent pathology reports, tumor recurrence identified on gadolinium-enhanced Magnetic Resonance Imaging (MRI), age 18-70 years, and a life expectancy of more than two months. In addition to undergoing surgery, all patients completed external-beam radiotherapy before being included in the study.

\section{The trial design and treatment administration}

Our study is a phase III randomized open label clinical trial with patients randomly assigned in a 1 to 1 ratio to receive either Specific Conjugate Particle Doxorubicin (SCP-Dox) or Pegylated Liposomal Doxorubicin (PEG-Dox) at a starting dose of $20 \mathrm{mg} / \mathrm{m}^{2}$ by an intravenous infusion over 30 minutes per day \{the regimen is not clear, which day is the therapy given ? how long is a cycle? Were there any maximal number of cycles to be given or until progression or until Grade 3 to 4 toxicity occured?\}. Doses were escalated by 40 $\mathrm{mg} / \mathrm{m}^{2}$ every fourth day until the total dose reached $480 \mathrm{mg}$ \{what is the maximum dose per body surface area\} or until Grade 3 or 4 toxicity occurred. If Grade 3 or 4 myelotoxicity occurred, the next chemotherapy cycle was delayed. In addition to chemotherapy, standard supportive care measures were applied to avoid super added infections.

\section{Evaluation of response and toxicity}

All patients underwent an MRI with gadolinium before study inclusion and before every fourth treatment course (every 6 weeks) to determine tumor response. When clinical deterioration was observed, a repeat MRI was performed. Clinical data, as well as Karnofsky Performance Status (KPS) and Mini-Mental Status, were assessed before each cycle. Standard laboratory parameters were measured each week or more frequently, if clinically necessary.

Progression-free survival at six months after the initiation of therapy (PFS-6) and toxicity was defined as the primary endpoints of the study; Progression-Free Survival at 12 months (PFS-12), Time to Progression (TTP) and Overall Survival (OS) were secondary endpoints. Established criteria were based on tumor response [22]. Side effects were graded according to the National Cancer Institute Common Toxicity Criteria (Version 4.0) for chemotherapy-related side effects.

\section{Statistics methods}

The researchers based the current study on an adapted intentto-treat design. Cox's Regression Model of life table analysis was 
Citation: Allen T, Khoni NS, Dunphy B, Kinge ARE, Court NB. Efficacy and safety of Specific Conjugate Particle (SCP)-Doxorubicinin Patients with Recurrent High-Grade Gliomas, a Randomized Clinical Study. J Vaccine Immunotechnology. 2017;3(1): 5.

ISSN: 2377-6668

Table 1: Demographic data.

\begin{tabular}{|l|l|l|l|l|l|}
\hline & $\begin{array}{l}\text { All } \\
\text { patients }\end{array}$ & $\begin{array}{l}\text { WHO grade } \\
\text { III }\end{array}$ & $\begin{array}{l}\text { WHO Grade } \\
\text { IV }\end{array}$ & PEG-Dox & SCP-Dox \\
\hline No. of patients & 80 & 24 & 56 & 40 & 40 \\
\hline Male & 56 & 16 & 40 & 24 & 32 \\
\hline Female & 24 & 8 & 16 & 16 & 8 \\
\hline Median age & $\begin{array}{l}49.5(27- \\
68)\end{array}$ & & & & \\
\hline
\end{tabular}

Table 2: Efficacy data [it is better to be presented as a column chart or keep the same with clear differentiation between the 2 groups.

\begin{tabular}{|l|l|l|}
\hline \multicolumn{2}{|c|}{ Efficacy data } \\
\hline All patients* & Responses & p-value \\
\hline WHO grade III & 32 & Not Significant (NS) \\
\hline WHO grade IV & 10 & NS \\
\hline WHO grade III vs. IV & 22 & NS \\
\hline PEG-Dox** & & NS \\
\hline SCP-Dox*** & 8 & As noted below \\
\hline
\end{tabular}

SCP-Dox vs. PEG-Dox response rate significantly higher $(p<0.05)$

*Response: Includes 2 complete response, 2 partial response, and 28 cases of stable disease.

${ }^{* *}$ Response PEG-Dox: 8 cases of stable disease.

${ }^{* \star *}$ Response SCP-Dox: Includes 2 complete response, 2 partial response, and 20 cases of stable disease.

utilized to assess outcomes and statistical significance was confirmed via Kaplan-Meier estimates. After four treatment courses (8 weeks), response status was determined for the first time. In clinical trials involving patients with high-grade glioma, it is common to count patients with Stable Disease (SD) as responders, as was done in the statistical analysis. Nonetheless, Complete Responses (CRs), Partial Responses (PRs), and cases of SD are reported separately in the text. Cox's regression method was used to estimate the risk of occurrence of defined events, and significance was assessed using the Wilcoxon test.

\section{Results}

\section{Patient characteristics}

Eighty patients (56 males and 24 females) with a recurrent high-grade glioma were recruited to the study and were available for assessment. These included 54 patients with glioblastoma, two patients with gliosarcoma, 22 patients with anaplastic astrocytoma, and two patients with anaplastic oligoastrocytoma. All patients had undergone open surgery at initial diagnosis, with the goal of maximum tumor removal, and received conventional whole brain irradiation .

A total of 563 courses of SCP-Dox/PEG-DOX were administered, with a range of 1-28 courses per patient. Limited issues were observed with SCP-Dox administration, and patients were evaluated for toxicity and response. Patient groups for PEG-Dox and SCP-Dox were homogeneous forage and extent of first-line resection (Table 1). In the SCP-Dox group, however, there were significantly more patients who had a Grade IV tumor (32, compared with 24 in PEGDox).

\section{Efficacy}

All patients were monitored until either tumor progression or death occurred, and no patients were lost to follow-up. In the 80 patients evaluated, the overall response rate was $40 \%$. We observed two complete responses (CRs) and two Partial Responses (PRs). We noted Stable Disease (SD) lasting longer than eight weeks in 28 patients, including 18 patients with Grade IV disease and ten patients with Grade III disease (Table 2). Patients receiving SCP-Dox had a significantly better response to therapy (more CR, PR, and SD) than those receiving PEG-Dox.

Comparison of the two treatment regimens revealed significant differences for Progression Free Survival (PFS) and Overall Survival (OS) as documented in (Table 3). PFS for SCP-Dox and PEG-Dox at 6 months was $75 \%$ and $25 \%$ respectively, and at 12 months was $50 \%$ and $12.5 \%$ respectively.

By definition, PFS at 6 months only provides data for the time point of 6 months after commencing therapy. However, it is being used increasingly in Phase III studies as it becomes clear that response rates are not a robust predictor of Time To Progression (TTP) or survival; PFS at 6 months, in contrast, corresponds well to median TTP.

\section{Safety}

Chemotherapy was discontinued in six patients on PEG-Dox but none on SCP-Dox. It was either due to toxicity (deep vein thrombosis in one patient) or at the patient's request to leave the trial. The most common adverse event observed was Palmoplantar Erythrodysesthesia (PPE) (grade 2-3), which was noted in 12 patients (2 receiving SCP-Dox and 10 receiving PEG-Dox). Two patients on PEG-Dox developed bullous exanthema \{? Grade 3\}, which resulted in treatment delay. Those patients who developed Palmoplantar Erythrodysesthesia were treated with oral pyridoxine and methyl prednisolone as required. Six patients on PEG-Dox developed mucositis (Grade 2) with superinfection, however, it was possible to

Table 3: Patients survival data (PFS=Progression Free Survival, OS=Overall Survival) [because there is clear difference, a line chart is much better to point the PFS and OS; i.e Kaplan-Meier curve.

\begin{tabular}{|c|c|c|c|}
\hline \multicolumn{4}{|c|}{ Patient survival data } \\
\hline Primary End Points & SCP-Dox & PEG-Dox & $P$ values \\
\hline $\begin{array}{l}\text { Progression Free } \\
\text { Survival at } 6 \text { months } \\
\text { (PFS-6) }\end{array}$ & $30(75 \%)$ & $10(25 \%)$ & $P<0.05$ \\
\hline \multicolumn{4}{|l|}{ Secondary Endpoints } \\
\hline $\begin{array}{l}\text { Progression Free } \\
\text { Survival at } 12 \text { months } \\
\text { (PFS-12) }\end{array}$ & $20(50 \%)$ & $5(12.5 \%)$ & $P<0.05$ \\
\hline \multicolumn{4}{|c|}{ Overall Survival from diagnosis (\%) } \\
\hline 6 months & $100 \%$ & $100 \%$ & \\
\hline 12 months & $100 \%$ & $75 \%$ & \\
\hline \multicolumn{4}{|c|}{ Overall Survival from recurrence (\%) } \\
\hline 6 months & $30(75 \%)$ & $10(25 \%)$ & \\
\hline 12 months & $26(65 \%)$ & $6(15 \%)$ & \\
\hline
\end{tabular}


Table 4: Safety data.

\begin{tabular}{|l|c|c|c|c|}
\hline Adverse events & \multicolumn{3}{|c|}{$\begin{array}{c}\text { SCP-Dox } \\
(n=40)\end{array}$} & \multicolumn{2}{|c|}{$\begin{array}{c}\text { PEG-Dox } \\
(n=40)\end{array}$} \\
\cline { 2 - 6 } & $\begin{array}{c}\text { Grade } \\
1-2\end{array}$ & $\begin{array}{c}\text { Grade } \\
3-4\end{array}$ & Grade 1-2 & $\begin{array}{c}\text { Grade } \\
3-4\end{array}$ \\
\hline Palmoplantar erythrodysesthesia & 2 & & 10 & \\
\hline Mucositis & 0 & & 6 & \\
\hline Superinfection & 1 & & 4 & \\
\hline Myelotoxicity & 1 & & 4 & \\
\hline Deep vein thrombosis & 0 & & 1 & \\
Total & 4 & & 25 & \\
\hline
\end{tabular}

continue therapy for those patients. In the group receiving PEG-Dox, serious myelotoxicity (Grade 3 or 4) was observed in three patients who had experienced Grade 3 myelotoxicity during the previous standard chemotherapy.

No cardio-toxic side effects were observed in either group (including cases where the maximum cumulative dose was $>400$ $\mathrm{mg} / \mathrm{m}^{2}$ ). One patient in PEG-Dox developed a deep vein thrombosis. No fatalities occurred as a result of treatment-related toxicity. As documented in (Table 4), a total of 25 adverse events occurred in patients receiving PEG-Dox, whereas there were only 4 adverse events in patients who received SCP-Dox. The most common serious adverse reactions observed conventional or liposomal doxorubicin are carditoxicity (48\%), mucositis (23\%), myelosupression (10\%), Palmarplantar Erythrodysesthesia (PPE)/hand-foot syndrome (48\%) [23].

\section{Discussion}

SCP-Dox was found to have superior efficacy to PEG-Dox in the management of recurrent malignant glioma, particularly in cases that had Grade III glioma. SCP-Dox also proved to be a safer treatment regimen, with no major side effects in our study population when compared to PEG-Dox.

Of note, non-responders were treated at considerably later stages of disease compared with responders ( 54 weeks vs. 35 weeks). Although this study was undertaken utilizing rigorous methodologies, there are some potential limitations. Firstly, the population of the experimental group is small, as it involved only eighty patients and so may not be representative of the general population. However, there was a statistically significant superior efficacy and reduced side effect profile associated with SCP-Dox. Secondly; patients were followed up for 12 months only. Future studies could undertake a longer period of follow-upbased on the stage of carcinoma. These limitations would suggest further research may be required to confirm the promising results of this study, which include a larger number of patients over a longer period.

Solubility performance is the most challenging feature for various new chemical entities. Almost $60 \%$ of the new potential products retain solubility complications. This is the main cause for some of the New Drug Applications which have not successfully entered the market or reached their full clinical potential. There are many techniques in attempting to improve the drug solubility. A hybrid method in utilizing particle size reduction, nano suspension, and the use of solid dispersion, are employed in producing SCP. Solid dispersion is an important approach for improvement of bioavailability of poor water-soluble drugs; however, our employed techniques of achieving SCPs are not unique to using a single hydrolyzing agent such as surfactants. A combination of the appropriate linking hydrophilic structure with the manufacturing techniques in achieving long shelf life of the produced structured SCPs is the key to its advantageous landscape. This is particularly important to combine the advanced technology of its production, with inexpensive product line, long shelf life, as well as its strong multi- aspect of IP protection. From technical and regulatory prospective as well as its legal front, SCP is a multi- edge advancement in drug delivery techniques.

\section{Conclusions and Future Directions}

However, to generalize the results, future research should involve more participants over a longer period of follow-up. We anticipate that improved results might be anticipated where patients are treated earlier in the course of their disease.

The development of SCP-Doxorubicin may be accompanied by a number of important lessons for the agent and for cancer therapy as a whole. These lessons have led to a successful clinical trial program for SCP-Dox in recurrent high grade gliomas. SCP-Dox is the first modulated Doxorubicin to demonstrate a statistically significant improvement in overall survival in a trial in patients with recurrent high grade gliomas. The predicted adverse events associated with SCP-Dox therapy are well described and reflect its small conjugated particle mechanism of action. Clinical studies showed that most adverse events were reversible using product-specific treatment guidelines, including prolonged duration of treatment and/or early temporary discontinuation of treatment. These guidelines can reduce the incidence of life-threatening events. The nature of the adverse events observed with SCP-Dox, along with potential safety studies, support the ability of SCP-Dox to break peripheral cytotoxic tolerance and to potentiate an antitumor chemotherapeutic response mediated by leaky tumor phenomenon. The kinetics of response and survival data for SCP-Dox in high grade gliomas are characterized based on new insights for small particle development and may have general applicability for similar therapies. Thus, the observations made during the development of SCP-Dox add to the growing evidence for the utility of the clinical paradigm for cancer cytotoxic therapies as may be defined by the Cancer Consortiums.

\section{Disclaimer Statement}

This press release contains forward-looking statements within the meaning of the Private Securities Litigation Reform Act of 1995, Section 27A of the Securities Act of 1933, as amended, and Section $21 \mathrm{E}$ of the Securities Exchange Act of 1934, as amended. Forwardlooking statements relate to Nexus Alliance Biopharmaceuticals (NAB) current expectations, beliefs, projections and similar expressions concerning matters that are not historical facts and are not guarantees of future performance. Forward-looking statements involve uncertainties, risks, assumptions and contingencies, many of which are outside NAB's control that may cause actual results to differ materially from those described in or implied by any forward-looking statements. All forward-looking statements are based on currently available information and speak only as of the date on which they 
Citation: Allen T, Khoni NS, Dunphy B, Kinge ARE, Court NB. Efficacy and safety of Specific Conjugate Particle (SCP)-Doxorubicinin Patients with Recurrent High-Grade Gliomas, a Randomized Clinical Study. J Vaccine Immunotechnology. 2017;3(1): 5.

are made. $\mathrm{NAB}$ assumes no obligation to update any forward-looking statement made in this press release that becomes untrue because of subsequent events, new information or otherwise, except to the extent it is required to do so in connection with its ongoing requirements under Federal securities laws. For a further discussion of factors that could cause NAB's future results to differ materially from any forward-looking statements, see the section entitled "Risk Factors" in NAB's Annual Report on Form 10-K for the year ended June 30, 2017 and other risks described in documents filed by NAB from time to time with the Securities and Exchange Commission or other notified financial bodies.

\section{References}

1. Ostrom QT, Gittleman H, de Blank PM, Finlay JL, Gurney JG, et al. (2016) American Brain Tumor Association adolescent and young adult primary brain and central nervous system tumors diagnosed in the United States in 20082012. Neuro Oncol 18: i1-i50

2. National Comprehensive Cancer network (2006) Central nervous system cancers. NCCN Clinical Practice Guidelines in Oncology, Version 2.

3. Glioma Meta-analysis Trialists (GMT) Group (2002) Chemotherapy for highgrade glioma. Cochrane Database Syst Rev 4: CD003913.

4. Stupp R, Hegi ME, Mason WP, van den Bent MJ, Taphoorn MJ, et al. (2009) Effects of radiotherapy with concomitant and adjuvant temozolomide versus radiotherapy alone on survival in glioblastoma in a randomised phase II study: 5-year analysis of the EORTC-NCIC trial. Lancet Oncol 10: 459-466.

5. Stupp R, Mason WP, van den Bent MJ, Weller M, Fisher B, et al. ( 2005) Radiotherapy plus concomitant and adjuvant temozolomide for glioblastoma N Engl J Med 352: 987-996.

6. Wolff JE, Trilling T, Molenkamp G, Egeler RM, Jurgens H (1999) Chemosensitivity of glioma cells in vitro: a meta-analysis. J Cancer Res Clin Oncol 125: 481-486.

7. Siegal T, Horowitz A, Gabizon A (1995) Doxorubicin encapsulated in sterically stabilized liposomes for the treatment of brain tumor model: biodistribution and therapeutic efficacy. J Neurosurg 83: 1029-1037.

8. Cabanes A, Tzemach D, Goren D, Horowitz AT, Gabizon A (1998) Comparative study of the antitumor activity of free doxorubicin and polyethylene glycol-coated liposomal doxorubicin in a mouse lymphoma model. Clin Cancer Res 4: 499-505.

9. Twentyman PR (1992) MDR1 (P-glycoprotein) gene expression--implications for resistance modifier trials. J Natl Cancer Inst 84: 1458-1460.

10. Cole SP, Deeley RG (1998) Multidrug resistance mediated by the ATPbinding cassette transporter protein MRP. Bioessays 20: 931-940.

11. Bredel M, Zentner J (2002) Brain-tumour drug resistance: the bare essentials Lancet Oncol 3: 397-406.

12. Abe T, Mori T, Wakabayashi Y, Nakagawa M, Cole SP, et al. (1998) Expression of multidrug resistance protein gene in patients with glioma after chemotherapy. J Neurooncol 40: 11-18.

13. Reya T, Morrison SJ, Clarke MF, Weissman IL (2001) Stem cells, cancer, and cancer stem cells. Nature 414: 105-111.

14. Dean M, Fojo T, Bates S (2005) Tumour stem cells and drug resistance. Nat Rev Cancer 5: 275-284

15. Jones RJ, Matsui WH, Smith BD (2004) Cancer stem cells: are we missing the target? J Natl Cancer Inst 96: 583-595.

16. Wang $Y$ (2011) Effects of salinomycin on cancer stem cell in human lung adenocarcinoma A549 cells. Med Chem 7: 106-111.

17. Kakarala M, Brenner DE, Korkaya H, Cheng C, Tazi K, et al. (2010) Targeting breast stem cells with the cancer preventive compounds curcumin and piperine. Breast Cancer Res Treat 122: 777-785.

18. Choi S, Lew KL, Xiao H, Herman-Antosiewicz A, Xiao D, et al. (2007) D,LSulforaphane-induced cell death in human prostate cancer cells is regulated by inhibitor of apoptosis family proteins and Apaf-1. Carcinogenesis 28: 151162

19. Jeong WS, Kim IW, Hu R, Kong AN (2004) Modulatory properties of various natural chemopreventive agents on the activation of NF-kappaB signaling pathway. Pharm Res 21: 661-670.

20. Moltzahn FR, Volkmer JP, Rottke D, Ackermann R (2008) "Cancer stem cells"-lessons from Hercules to fight the Hydra. Urol Oncol 26: 581-589.

21. Macdonald DR, Cascino TL, Schold SC Jr, Cairncross JG (1990) Response criteria for phase II studies of supratentorial malignant glioma. J Clin Oncol 8: $1277-1280$.

22. O'Brien ME, Wigler N, Inbar M, Rosso R, Grischke E, et al. (2004) Reduced cardiotoxicity and comparable efficacy in a phase III trial of pegylated liposomal doxorubicin $\mathrm{HCl}$ (CAELYX/Doxil) versus conventional doxorubicin for first-line treatment of metastatic breast cancer. Ann Oncol 15: 440-449 